\title{
In vitro screening of Kluyveromyces strains isolated from Fiore Sardo cheese for potential use as probiotics
}

\author{
Maria Elisabetta Fadda, Valentina Mossa, Maura Deplano, Maria Barbara Pisano, \\ Sofia Cosentino* \\ Department of Public Health, Clinical and Molecular Medicine, University of Cagliari, Cittadella Universitaria, SS 554, 09042, Monserrato (CA), Italy
}

\section{A R T I C L E I N F O}

\section{Article history:}

Received 9 June 2016

Received in revised form

26 July 2016

Accepted 13 August 2016

Available online 16 August 2016

\section{Keywords:}

Kluyveromyces

Probiotics

Fiore Sardo

Molecular characterization

\begin{abstract}
A B S T R A C T
In this work, six Kluyveromyces strains isolated from artisanal Fiore Sardo cheese were evaluated for some functional properties relevant to their use as probiotics.

All strains were able to grow in the presence of conjugated bile salts after $72 \mathrm{~h}$ of incubation, and four were able to hydrolyse both sodium glycodeoxycholate and taurodeoxycholate. All strains survived well under simulated gastric conditions $(\mathrm{pH} 3.0)$, but Kl. marxianus strains presented the best survival rates values (83-100\%) after exposure to artificial duodenum juice. Autoaggregation ability showed a certain variability with mean values ranging between 39.1 and 59.2\%, while the hydrophobicity index was higher than $50 \%$ in five strains. All strains were able to adhere to Caco-2 cells (values ranging from 4 to 68\%), with two Kl. lactis strains exhibiting significantly higher percentage adhesion than S. boulardii Codex used as control $(\mathrm{P}<0.05)$. Kl. lactis strains also showed the broadest inhibitory range against pathogens. Finally, all strains were non-hemolytic and sensitive to the antimycotic agents tested.

In conclusion, our results indicate that Kluyveromyces strains isolated from artisanal cheese possess interesting functional traits and absence of undesirable properties, and could be considered appropriate probiotic candidates.
\end{abstract}

๑) 2016 Elsevier Ltd. All rights reserved.

\section{Introduction}

In the last years, increasing attention has been devoted to the development of functional foods containing probiotic microorganisms for health promotion and disease prevention in the host.

Probiotics are defined as "live microorganisms which upon ingestion in adequate amounts confer a health benefit to the host" (FAO/WHO, 2006). Most probiotics are bacteria, mainly belonging to the genera Lactobacillus and Bifidobacterium (Gueimonde \& Salminen, 2006), while the use of yeasts is still limited, in spite of their widespread occurrence in several fermented food products, where they play an active role in production of aroma components and inhibition against spoilage microorganisms (Fleet, 2007). To our knowledge only the yeasts Saccharomyces boulardii and Kluyveromyces marxianus fragilis are commercialized as probiotic for humans, having been proven effective in clinical studies (Czerucka, Piche, \& Rampal, 2007; Maccaferri, Candela, Centanni, Severgnini,

\footnotetext{
* Corresponding author. Tel.: +39 0706754146; fax: +39 0706754197.

E-mail address: scosenti@unica.it (S. Cosentino).
}

Consolandi, 2012), but some authors have suggested the use of other yeast species or genera based essentially on their ability to survive during the passage through the human gastro-intestinal tract, their tolerance to low $\mathrm{pH}$ and bile salts, and their capability to control pathogenic microorganisms (Moslehi-Jenebian, Pedersen, \& Jespersen, 2010). Therefore, studies focusing on selecting new probiotic yeast strains isolated from food in which their occurrence is well known could be of interest.

Fiore Sardo is a typical Sardinian hard cheese, produced exclusively in Sardinia according to ancient production techniques. It is a protected designation of origin cheese made from raw ewe's milk without the addition of starter cultures, and therefore the maturation period is carried out solely by the indigenous microbial flora present in the milk and in the dairy environment. In a previous survey carried out in our laboratory to identify the yeast species present throughout the ripening process of this artisanal ewe's cheese, several autochthonous yeast strains, characterized both technologically and genotypically, showed particular properties which can contribute to the production of typical cheese taste and flavor (Fadda, Mossa, Pisano, Deplano, \& Cosentino, 2004). Among the predominant species identified, Kluyveromyces lactis and 
Kluyveromyces marxianus are included in the European Food Safety Authority list of Qualified Presumption of Safety biological agents added to food and feed (EFSA, 2013). Since the importance of these species in food fermentation is well known, characterization of their probiotic properties is gaining relevance.

The aim of this work was to evaluate six Kluyveromyces strains for some functional properties relevant to their use as probiotic cultures.

\section{Materials and methods}

\subsection{Yeast strains}

The yeast strains (Table 1) were isolated from Fiore Sardo cheese as previously reported (Fadda et al., 2004) and maintained in our laboratory's culture collection. The probiotic strain Saccharomyces boulardii isolated from the commercial product Codex (Zambon, Italy) and a strain of Kluyveromyces lactis isolated from a milky kefir grain obtained from a family that traditionally consumed kefir, were included in the study for comparison purpose. All strains were able to grow at $37^{\circ} \mathrm{C}$.

\subsection{Molecular characterization of strains}

\subsubsection{PCR-RFLP analysis}

Genomic DNA was extracted as previously described (Fadda, Viale, Deplano, Pisano, \& Cosentino, 2010). NS1 (5'-GTAGTCATATGCTTGTCTC- $3^{\prime}$ ) and ITS2 (5'-GCTGCGTTCTTCATCGATGC-3') primers were used to amplify most of the $18 \mathrm{~S}$ gene, the ITS1 segment and most of the 5.8S gene in ribosomal DNA. Amplification and digestion of PCR products with HaeIII (Sigma, Milano, Italy) were carried out according to Fadda et al. (2013).

Dendrogram analysis of RFLP fragments was carried out with GelComparII software package, version 6.5 (Applied Math, Kortrijk, Belgium), using the Unweighted Pair-Group Method with Arithmetic mean (UPGMA) algorithm and Dice similarity coefficient. Restriction fragments shorter than 100 bp were not considered in the analysis.

\subsubsection{DNA sequencing}

The D1/D2 domain of the 26S rRNA gene was amplified and sequenced as previously described (Fadda et al., 2013). The sequences are deposited at GenBank National Center for Biotechnology Information (NCBI), USA under the accession numbers listed in Table 1.

\subsection{Probiotic evaluation}

\subsubsection{Bile salt hydrolase activity}

Bile salt hydrolase (BSH) activity was screened by spotting in duplicate $10 \mu \mathrm{L}$ of cultures grown overnight in yeast-extract-

\section{Table 1}

Origin and molecular identification of dairy yeast strains by sequencing of the domains D1/D2 of the subunit 26S rRNA gene.

\begin{tabular}{llll}
\hline Strain & Origin & Molecular identification & Accession GenBank \\
\hline CODEX SB1 & Codex & S. boulardii & K. lactis \\
KEFIR KL1 & Kefir & K. lactis & KP288823 \\
17bKL1 & Fiore Sardo & K. lactis & KP288824 \\
17bKL2 & Fiore Sardo & K. lactis & KP288825 \\
NS2KL9 & Fiore Sardo & K. lactis & KP268080 \\
NS1KM2 & Fiore sardo & K. marxianus & KP268079 \\
14KM1 & Fiore Sardo & K. marxianus & KP268081 \\
6688 KM & Fiore Sardo & K. marxianus & \\
\hline
\end{tabular}

peptone-dextrose (YEPD, Microbiol, Italy) broth on the surface of YEPD agar plates supplemented with $0.5 \%(\mathrm{w} / \mathrm{v})$ sodium salt taurodeoxycholic acid (TDCA, Sigma) or $0.2 \%(\mathrm{w} / \mathrm{v})$ glycodeoxycholic acid (GDCA, Sigma) and $0.37 \mathrm{~g} \mathrm{~L}^{-1}$ of $\mathrm{CaCl}_{2}$. Plates were incubated at $37{ }^{\circ} \mathrm{C}$ for $72 \mathrm{~h}$. The presence of halos around colonies (in YEPDGDCA) or white opaque colonies (in YEPD-TDCA) indicated BSH activity.

\subsubsection{In vitro resistance to gastro-intestinal conditions (SSDP test)}

The strains were inoculated to a final concentration of approximately $5 \times 10^{6}$ colony forming unit (cfu) $\mathrm{mL}^{-1}$ in $10 \mathrm{~mL}$ of simulated gastric juice $\left(6.2 \mathrm{~g} \mathrm{~L}^{-1} \mathrm{NaCl}, 2.2 \mathrm{~g} \mathrm{~L}^{-1} \mathrm{KCl}, 0.22 \mathrm{~g} \mathrm{~L}^{-1} \mathrm{CaCl}_{2}\right.$, $1.2 \mathrm{~g} \mathrm{~L}^{-1} \mathrm{NaHCO}_{3}, 0.3 \%$ pepsin, $\mathrm{pH} 3.0$ ) and incubated at $37^{\circ} \mathrm{C}$ in a shaking water bath (Dubnoff 750 , Asal, Italy), to simulate peristalsis. After $90 \mathrm{~min}, 17.5 \mathrm{~mL}$ of synthetic duodenum juice $\left(6.4 \mathrm{~g} \mathrm{~L}^{-1}\right.$ $\mathrm{NaHCO}_{3}, 0.239 \mathrm{~g} \mathrm{~L}^{-1} \mathrm{KCl}, 1.28 \mathrm{~g} \mathrm{~L}^{-1} \mathrm{NaCl}, 0.1 \%$ pancreatin) adjusted to $\mathrm{pH} 7.4$ with $5 \mathrm{M} \mathrm{HCl}$, and $4 \mathrm{~mL}$ of $10 \%$ (w/v) oxgall (Sigma) were added to the cell suspensions to simulate passage into the upper intestinal tract (Pisano et al., 2014). After 0 (T0), 90 (T1), and 270 (T2) min of incubation, the survival rate was determined by the plate method using YEPD incubated at $37^{\circ} \mathrm{C}$ for $48 \mathrm{~h}$.

\subsubsection{Autoaggregation capacity}

Overnight yeast cultures in YEPD broth were centrifuged for $10 \mathrm{~min}$ at $5000 \mathrm{~g}$ at $4{ }^{\circ} \mathrm{C}$, washed twice and resuspended in phosphate buffered saline (PBS, $3 \mathrm{~mL}$ ), vortexed for $30 \mathrm{~s}$ and incubated for $2 \mathrm{~h}$ at $37^{\circ} \mathrm{C}$. An aliquot ( $1 \mathrm{~mL}$ ) of these suspensions was carefully removed from the upper zone, and the $\mathrm{OD}_{560 \mathrm{~nm}}$ was measured before and after incubation, using a spectrophotometer (Ultraspec 3000, Pharmacia Biotech, USA). The autoaggregation was expressed as $1-\left(\mathrm{ODt} / \mathrm{OD}_{0}\right) \times 100$, where $\mathrm{OD}_{0}$ and ODt are the optical density before and after incubation, respectively.

\subsubsection{Hydrophobicity}

Overnight yeast cultures in YEPD broth were centrifuged for $10 \mathrm{~min}$ at $5000 \mathrm{~g}$ at $4{ }^{\circ} \mathrm{C}$, washed twice and resuspended in PBS adjusted to an $\mathrm{OD}_{560 \mathrm{~nm}}$ of approximately 1.0 in the same buffer. Three milliliters of these suspensions were added to $0.6 \mathrm{~mL}$ of $\mathrm{n}$ hexadecane (Sigma) and vortexed for $120 \mathrm{~s}$. The two phases were allowed to separate for $1 \mathrm{~h}$ at $37^{\circ} \mathrm{C}$. The aqueous phase was carefully removed and the $\mathrm{OD}_{560 \mathrm{~nm}}$ was measured. The percentage of hydrophobicity was evaluated as the percentage of reduction in the optical density of the aqueous phase and calculated with the formula $\left[\left(\mathrm{OD}_{0}-\mathrm{OD}\right) / \mathrm{OD}_{0}\right] \times 100$, where $\mathrm{OD}_{0}$ and $\mathrm{OD}$ are the optical density before and after extraction with n-hexadecane, respectively.

\subsubsection{Adhesion to Caco-2 cells}

The human colonic carcinoma cell line Caco-2 (ECACC, Salisbury, UK) was routinely cultured in Dulbecco's modified Eagle's minimal essential medium (DMEM, Sigma), supplemented with $10 \%(\mathrm{w} / \mathrm{v})$ fetal bovine serum (FBS, Sigma), 1\% (w/v) nonessential amino acids solution (Sigma), and antibiotic solution (100 $\mathrm{U} \mathrm{mL}^{-1}$ penicillin, $100 \mu \mathrm{g} \mathrm{mL}^{-1}$ streptomycin). Cells were maintained in T-75 culture flasks at $37{ }^{\circ} \mathrm{C}$ in a $5 \% \mathrm{CO}_{2}$ atmosphere. For adhesion assay, the Caco-2 cells were seeded at a concentration of $10^{5}$ cells/well in 6well tissue culture plates (Falcon ${ }^{\circledR}$, Corning, USA) to obtain confluence and cultured for 14 days prior to use in adhesion assay. The cell culture medium was changed on alternate days and replaced by fresh DMEM supplemented with $2 \%(w / v)$ FBS and without antibiotic at least $1 \mathrm{~h}$ before the adhesion assay. A $1 \mathrm{~mL}$ aliquot of yeast suspension $\left(10^{6} \mathrm{cfu} \mathrm{mL}^{-1}\right.$ in PBS) was added to each well of the tissue culture plate and incubated at $37{ }^{\circ} \mathrm{C}$ in a $5 \% \mathrm{CO}_{2}$ atmosphere for $3 \mathrm{~h}$. Afterwards, the cells were washed three times with $1 \mathrm{~mL}$ of PBS in order to remove non adherent yeasts and lysed 
by addition of Triton X-100 (0.05\% solution) for $10 \mathrm{~min}$, then appropriate dilutions were plated on YEPD agar. Adhesion was expressed as the percentage of yeast adhered to Caco-2 cells compared to the initial amount.

\subsubsection{Antimicrobial activity}

The strains were screened for antagonist activity against the indicator strains listed in Table 5 using the agar spot method described by Schillinger and Lücke (1989) with some modifications. Fresh overnight cultures of yeast strains were spotted onto the surface of YEPD agar plates, incubated for $48 \mathrm{~h}$ at $37^{\circ} \mathrm{C}$. The indicator strains were inoculated into $7 \mathrm{~mL}$ of soft agar medium (nutrient broth containing $0.7 \% \mathrm{w} / \mathrm{v}$ agar) to a final concentration of approximately $10^{7} \mathrm{cfu} \mathrm{\textrm {mL } ^ { - 1 }}$, then the soft media were poured on the plates. After $24 \mathrm{~h}$ of incubation at the optimal growth temperature and atmosphere for the indicator strains, inhibition halos were measured. The width of the clear zone $(R)$ was calculated as follows: $R=(d$ Inhib $-d S$ pot $) / 2$, where $d$ Inhib is the diameter of the zone without pathogen growth and $d$ Spot is the diameter of the spot.

\subsection{Safety assessment}

Hemolytic activity was determined by inoculating the strains on blood agar plates (Microbiol) containing 5\% defibrinated sheep blood after $48 \mathrm{~h}$ of incubation at $37^{\circ} \mathrm{C}$. The development of a clear zone of hydrolysis around the colonies was considered as positive result ( $\beta$-hemolysis).

The susceptibility of the strains to antifungal agents was assessed using the disc diffusion method according to CLSI document M44-A2 (2009). A suspension from fresh overnight cultures with a density of McFarland 0.5 was cultured on Mueller-Hinton agar plates supplemented with $2 \%$ glucose and $0.5 \mu \mathrm{g}$ of Methylene blue per ml. Filter paper discs (Rosco Diagnostica, Denmark) with different antimycotics were placed on the agar. After $24 \mathrm{~h}$ of incubation, the zones of inhibition around the discs were measured and the yeast strains were considered susceptible (S), intermediate (I) or resistant $(\mathrm{R})$ as reported in Table 6 .

\subsection{Statistical analysis}

All experiments were run in triplicate and data expressed as mean \pm standard deviation or standard error. Counts were con-

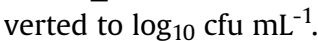

Statistical analysis of data was carried out using GraphPad Prism Statistics software package version 3.00 (GraphPad Prism Software Inc., San Diego, USA). One-way ANOVA followed by a Tukey's posthoc was used to determine significant differences of viability of the yeast strains during SSDP treatment and with respect to the adhesion ability. Differences were considered statistically significant at $P<0.05$.

\section{Results and discussion}

\subsection{Molecular characterization of strains}

Fig. 1 shows the PCR-RFLP profiles and clusters of the yeast strains analyzed. At a similarity of $65 \%$ the strains grouped in three clusters. Isolates of cluster 1 were identified by sequencing of the D1/D2 domain of the 26S rDNA as Kluyveromyces marxianus, while those of cluster 3 as Kluyveromyces lactis. Cluster 2 was represented by the Codex Saccharomyces boulardii strain. Kl. lactis from kefir was grouped with the other $\mathrm{Kl}$. lactis strains at a similarity value of $75 \%$.

In recent years, PCR-based molecular methods have been proved useful for yeast identification and characterization (El-Sharoud, Belloch, Peris, \& Querol, 2009; Fadda et al., 2013). Our results confirmed that PCR-RFLP analysis provides good discrimination at both species and strain level.

\subsection{Probiotic evaluation}

The main goal of this work was to study some functional properties of Kluyveromyces strains with the intention to propose their use as potential probiotics in foods.

To this purpose, 21 Kluyveromyces strains previously isolated from Fiore Sardo cheese and characterized by phenotypic and technological criteria (Fadda et al., 2004) were analyzed. Because tolerance to low $\mathrm{pH}$ and bile salts is considered a prerequisite for probiotic organisms to exert their beneficial effects, a preliminary screening based on the ability to growth at $\mathrm{pH} 2.0$ and in presence of bile salts was applied to the strains. Only six strains showed a percentage survival higher than 95\% after exposure to $\mathrm{pH} 2.0$ and in presence of $0.3 \%(\mathrm{w} / \mathrm{v})$ bile salts and were therefore selected for further study (data not shown).

BSH activity is a relevant property for probiotic strains to survive the toxicity of conjugated bile salts in the duodenum (de Smet, van Hoorde, van de Woestyne, Christiaens, \& Verstraete, 1995). In our study, all strains were able to grow in the presence of conjugated bile salts after $72 \mathrm{~h}$ of incubation, and five (Codex, 17bKL1, 17bKL2, NS2KL9, NS1KM2) also demonstrated the ability to hydrolyze both sodium glycodeoxycholate and sodium taurodeoxycholate, as indicated by the BSH test on agar plates (see Table 2). Some authors have shown that in yeasts the resistance to bile salts toxicity is not related to hydrolase activity (Aloglu, Ozer, \& Oner, 2016; Sourabh, Kanwar, \& Sharma, 2011). Other mechanisms, alternative to BSH, could be involved in counteracting bile damage, as suggested by

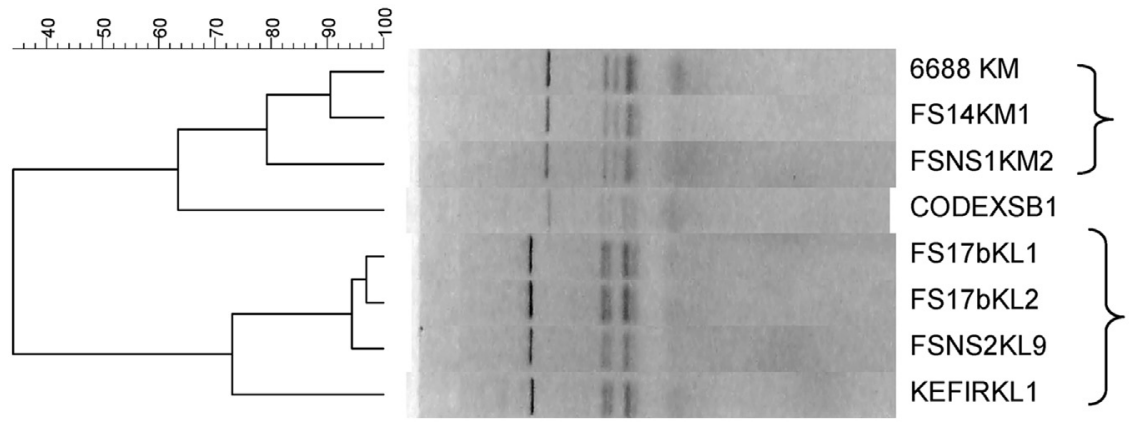

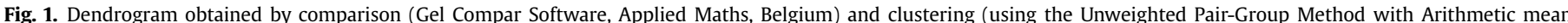

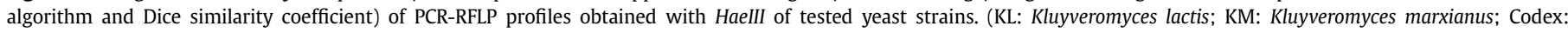
Saccharomyces cerevisiae). 
Table 2

Bile salt deconjugation activity of yeast strains on YEPD agar plates supplemented with $0.5 \%(\mathrm{w} / \mathrm{v})$ sodium salt taurodeoxycholic acid (TDCA) or $0.2 \%(\mathrm{w} / \mathrm{v})$ glycodeoxycholic acid (GDCA) and $0.37 \mathrm{~g} \mathrm{~L}^{-1}$ of $\mathrm{CaCl}_{2}$. (KL: Kluyveromyces lactis; KM: Kluyveromyces marxianus; Codex: Saccharomyces cerevisiae).

\begin{tabular}{llll}
\hline Strain & Growth & Hydrolysis of & \\
\cline { 2 - 4 } & & TDCA $^{\mathrm{a}}$ & GDCA $^{\mathrm{b}}$ \\
\hline CODEX SB1 & + & + & + \\
KEFYR KL1 & + & - & - \\
17bKL1 & + & + & + \\
17b KL2 & + & + & + \\
NS2KL9 & + & + & + \\
NS1KM2 & + & + & + \\
FS14KM1 & + & - & - \\
6688KM & + & - & - \\
\hline
\end{tabular}

(-) no activity.

a + : presence of halos around the colony was detected.

b + : white opaque colonies were observed.

Noriega, Cuevas, Margolles, and de los Reyes-Gavilán (2006). BSH activity is frequently observed in specific groups of bacteria like Lactobacillus and Bifidobacterium isolated from the gastro-intestinal tract (GIT) (Vizoso Pinto, Franz, Schillinger, \& Holzapfel, 2006) but it has rarely been reported for yeasts. In the study by Sourabh et al. (2011) none of the 23 S. cerevisiae isolates was able to show deconjugation activity on PDA plates supplemented with glycine and taurine conjugated bile salts while Aloglu et al. (2016) found several yeast strains of food origin with this property.

Presently the role of BSH is controversial since it has been reported to act either positively in lowering of serum cholesterol (Nguyen, Kang, \& Lee, 2007) or negatively in increasing the level of undesirable deconjugated bile salts (Corzo \& Gilliland, 1999). However, it is known that the bacteria most frequently used as probiotics (bifidobacteria and lactobacilli) cannot convert the unconjugated bile acids into secondary bile acids (Ramasamy, Abdullah, Wong, Karuthan, \& Ho, 2010). On the other hand, BSH activity by a probiotic bacterium may be desirable since it increases the intestinal survival and persistence of producing strains, which in turn increases the overall beneficial effects associated with the strain (Begley, Hill, \& Gahan, 2006).

Potential probiotic yeasts should be able to survive while passing through the human GIT since viability plays an important role in certain of their beneficial properties (Romanin et al., 2010), therefore the strains were assessed in vitro for their survival after 90 and $270 \mathrm{~min}$ of exposure to different gastrointestinal conditions, as shown in Table 3.

All strains survived well under simulated gastric conditions $(\mathrm{pH}$ 3.0), which is not surprising since it could be related to an adaptation to acidic conditions in the natural environment (cheese) from which the strains were isolated and where they usually coexist with lactic acid bacteria. A reduction in viability was found for the Kl. lactis strains after exposure to artificial duodenum juice (pH 8.0), with the exception of the Kefir strain. Four strains maintained elevated cell numbers under conditions simulating passage through the human GIT, with values significantly higher $(\mathrm{P}<0.05)$ than those obtained for the probiotic strain S. boulardii Codex. Two $\mathrm{Kl}$. marxianus strains (FS14KM1 and $6688 \mathrm{KM}$ ) showed survival rates above $100 \%$ after $3 \mathrm{~h}$ despite the high bile concentration (more than $1 \%$ ) used in the test. These results are consistent with those previously reported for potentially probiotic yeast strains associated with food (Aloglu et al., 2016; Kumura, Tanoue, Tsukahara, Tanaka, \& Shimazaki, 2004), although the ability to survive under simulated GIT conditions has been reported to be variable and strain dependent in isolates of food origin (Binetti, Carrasco, Reinheimer, \& Suarez, 2013). Pennacchia, Blaiotta, Pepe, and Villani (2008) established a survival percentage benchmark $\geq 70 \%$ to select yeast strains as potential probiotics. In our study only four strains met this criterion, anyway all strains decreased their counts of about one log maximum, demonstrating they could reach the intestine in high numbers. Surprisingly, probiotic Codex strain showed a $64 \%$ decrease in viability after exposure to artificial duodenum juice, in contrast with the results of Perricone, Bevilacqua, Corbo, and Sinigaglia (2014) where S. boulardii ATCC-MYA-796 did not undergo any significant change in cell counts in bile salts and pancreatic juice. On the other hand, another strain of $S$. boulardii isolated from a commercial product was found to be more sensitive to bile salts than several kefir strains by Diosma, Romanin, ReyBurrusco, Londero, and Garrote (2014). These diversities may be due to different assay conditions in the studies.

Among the desirable properties of a potential probiotic microorganism is the ability to adhere to intestinal cells, thus providing advantages in the colonization of the GIT. Adhesion is a complex trait that could be a multistep process in which both non-specific and specific mechanisms play a role (Sourabh et al., 2011). One of the traits associated with the adhesion capacity is the ability to form cellular aggregates (Del Re, Sgorbati, Miglioli, \& Palenzona, 2000). As reported in Table 4, the autoaggregation percentages varied among the tested strains, with $S$. boulardii Codex displaying the highest value (81.5\%). The other strains showed intermediate autoaggregation ability with mean values between 39.1 and $59.2 \%$. Overall, Kl. lactis strains had percentages slightly higher than $\mathrm{Kl}$. marxianus strains, ranging from 44.5 to 59.2.

The isolates were also screened for their cell surface hydrophobicity property, that is considered to be another important factor in the adhesion and proliferation of microorganisms on the

Table 3

Resistance of yeast strains in simulated gastric and intestinal environment. All result are expressed as $\log _{10} \mathrm{cfu} \mathrm{mL}^{-1}$ ( $\mathrm{mean} \pm \mathrm{standard} \mathrm{deviation} \mathrm{of}$ three experiments). (KL: Kluyveromyces lactis; KM: Kluyveromyces marxianus; Codex: Saccharomyces cerevisiae).

\begin{tabular}{lllll}
\hline Strains & Initial mean count (T0) & $\begin{array}{l}\text { Survival after 90 min exposure } \\
\text { to artificial gastric juice (T1) }\end{array}$ & $\begin{array}{l}\text { Survival after 270 min of which } 180 \\
\text { in synthetic duodenum juice (T2) }\end{array}$ & $\begin{array}{c}\text { Decrease in viability \% } \\
\text { ind }\end{array}$ \\
\hline CODEXSB1 & $6.74 \pm 0.06$ & $6.62 \pm 0.03$ & $6.29 \pm 0.13$ & 64.5 \\
KEFYRKL1 & $6.60 \pm 0.15$ & $6.86 \pm 0.13$ & $6.54 \pm 0.28$ & 13.1 \\
17bKL1 & $6.99 \pm 0.39$ & $7.02 \pm 0.45$ & $6.49 \pm 0.01$ & 68.1 \\
17bKL2 & $6.79 \pm 0.07$ & $6.87 \pm 0.03$ & $6.14 \pm 0.09$ & 87.3 \\
NS2KL9 & $6.86 \pm 0.06$ & $6.73 \pm 0.09$ & $5.96 \pm 0.25$ & 17 \\
NS1KM2 & $6.75 \pm 0.06$ & $6.91 \pm 0$ & $6.67 \pm 0.15$ & 0 \\
FS14KM1 & $6.75 \pm 0.07$ & $6.79 \pm 0.19$ & $6.81 \pm 0.01$ & 0 \\
6688 KM & $6.91 \pm 0.02$ & $7.03 \pm 0.06$ & $6.96 \pm 0.14$ & \\
\hline
\end{tabular}

Artificial gastric juice contains $6.2 \mathrm{~g} \mathrm{~L}^{-1} \mathrm{NaCl}, 2.2 \mathrm{~g} \mathrm{~L}^{-1} \mathrm{KCl}, 0.22 \mathrm{~g} \mathrm{~L}^{-1} \mathrm{CaCl}_{2}, 1.2 \mathrm{~g} \mathrm{~L}^{-1} \mathrm{NaHCO}_{3}, 0.3 \%$ pepsin, $\mathrm{pH} 3.0$.

Synthetic duodenum juice contains $6.4 \mathrm{~g} \mathrm{~L}^{-1} \mathrm{NaHCO}_{3}, 0.239 \mathrm{~g} \mathrm{~L}^{-1} \mathrm{KCl}, 1.28 \mathrm{~g} \mathrm{~L}^{-1} \mathrm{NaCl}, 0.1 \%$ pancreatin, $4 \mathrm{~mL}$ of $10 \%$ (w/v) oxgall (adjusted to pH 7.4 with $5 \mathrm{M} \mathrm{HCl}$ )

${ }^{\mathrm{a}}$ Decrease in viability \% $=\left[\left(\mathrm{cfu} \mathrm{mL} \mathrm{L}^{-1} \mathrm{To}-\mathrm{cfu} \cdot \mathrm{mL}^{-1} \mathrm{T2}\right) / \mathrm{cfu} \mathrm{mL}^{-1} \mathrm{To}\right] \times 100$. 
Table 4

Auto-aggregation ( $\mathrm{Au}$ ) ability and hydrophobicity ( $\mathrm{HI}$ ) of yeast strains. Results are expressed as mean value \pm standard deviation of three determinations. (KL: Kluyveromyces lactis; KM: Kluyveromyces marxianus; Codex: Saccharomyces cerevisiae).

\begin{tabular}{lll}
\hline Strains & Auto-aggregation capacity $(\% \mathrm{Au})^{\mathrm{a}}$ & $\begin{array}{l}\text { Hydrophobicity } \\
\text { Index }(\% \mathrm{HI})^{\mathrm{b}}\end{array}$ \\
\hline CODEXSB1 & $81.5 \pm 0.03$ & $55.9 \pm 0.23$ \\
KEFYRKL1 & $42.5 \pm 0.08$ & $74.1 \pm 0.03$ \\
17bKL1 & $44.5 \pm 0.16$ & $39.9 \pm 0.05$ \\
17bKL2 & $59.2 \pm 0.11$ & $53.6 \pm 0.02$ \\
NS2KL9 & $54.6 \pm 0.03$ & $79.4 \pm 0.06$ \\
NS1KM2 & $52.8 \pm 0.02$ & $75.9 \pm 0.12$ \\
14KM1 & $42.9 \pm 0.04$ & $59.1 \pm 0.07$ \\
6688 KM & $39.1 \pm 0.04$ & $54.7 \pm 0.11$ \\
\hline
\end{tabular}

a Expressed as $1-\left(\mathrm{ODt} / \mathrm{OD}_{0}\right) \times 100$.

b Calculated with the formula $\left[\left(\mathrm{OD}_{0}-\mathrm{OD}\right) / \mathrm{OD}_{0}\right] \times 100$.

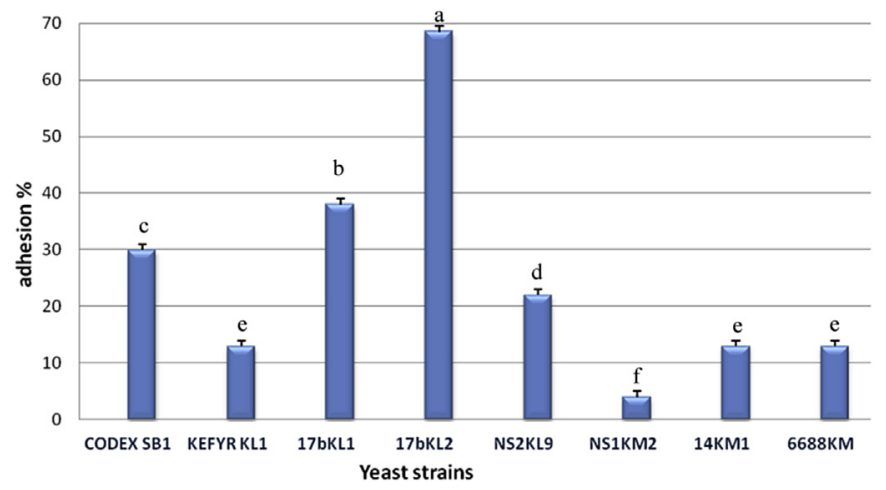

Fig. 2. Adhesion ability to Caco-2 human colon cell lines of dairy yeast strains (mean \pm ES of three independent experiments). Mean values with different superscript letters were significantly different at $\mathrm{P}<0.05$. Adhesion is expressed as the percentage of yeast adhered to Caco-2 cells compared to the initial amount. (KL: Kluyveromyces lactis; KM: Kluyveromyces marxianus; Codex: Saccharomyces cerevisiae).

intestinal epithelial cells (Del Re et al., 2000). Again, a certain variability was observed in our strains (Table 4), but for all isolates but one (17bKL1, 39.9\%) the values were above 50\%, ranging from 53.6 to $79.6 \%$.

In the work by Al-Seraih, Flahaut, Krier, Cudennec, and Drider (2015) the control strain S. boulardii IS showed autoaggregation and hydrophobicity values of 39.01 and 71.31, respectively, in good agreement with those obtained in our study for Codex strain. Our data are also in agreement with those reported by Binetti et al. (2013) for Kluyveromyces strains, and similarly we could not find a clear correlation between autoaggregation and hydrophobicity. In fact, as reported by other authors (Del Re et al., 2000; Rahman, Kim, Kumura, \& Shimazaki, 2008), although these traits are associated with adhesion they are independent of each other.

The ability to colonize the intestine, at least temporarily, by adhering to the intestinal epithelium has been indicated as a criterion to select probiotic yeasts (Tuomola, Crittenden, Playne, Isolauri, \& Salminen, 2001). The adhesive capacity of the yeast strains was evaluated using Caco-2 cells, a largely accepted in vitro model, and is reported in Fig. 2. All strains were able to adhere to Caco- 2 cells to various extents (ranging from 4 to 68\%), confirming that adhesion is a strain-specific property. Two Kl. lactis strains showed significantly higher adherence to Caco-2 cells than S. boulardii Codex used as positive control $(\mathrm{P}<0.05)$ and the other strains tested. Kl. lactis 17bKL2 was the most adhesive, since approximately $68 \%$ of the added bacteria were bound to Caco2 cells, followed by $\mathrm{Kl}$. lactis 17bKL1 and NS2KL9 (30 and 22\%, respectively). A lower adhesion rate was observed for Kl. marxianus strains (4-13\%). These findings are in agreement with those of other authors, who have observed the adherence ability of Kl. lactis strains and the inability of $S$. boulardii strains to adhere in in vitro experiments (Diosma et al., 2014; Kumura et al., 2004). In contrast, in the study by Maccaferri and Klinder et al. (2012) a dairy $\mathrm{Kl}$. marxianus strain (BO399) was classified as strongly adhesive.

Although probiotic adhesion to intestinal mucosa has been shown to interfere with the adherence of pathogens through competitive exclusion mechanisms (Jankowska, Laubiz, Antushevich, Zabielski \& Grzesiuk., 2008), it has been observed that even yeast strains with low adherence ability could be effective in reducing adhesion of pathogenic bacteria. van der Aa Kühle, Skovgaard and Jespersen (2005) demonstrated that low-adhering food-borne $S$. cerevisiae strains had protective effects on bacterial infections via reduction of the intestinal pro-inflammatory response, and Tiago et al. (2012) showed that some S. boulardii and S. cerevisisae strains were able to prevent Salmonella and E. coli adhesion to specific receptors on the intestinal epithelium, hence their invasion in the host, by capturing pathogens on their surface.

Antagonistic activity against pathogenic bacteria is among the important features of probiotic strains. As shown in Table 5, Kl. lactis strains showed the broadest inhibitory range, comparable to that of Codex: they were able to inhibit the growth of S. aureus ATCC 25923 with clear inhibition zones of more than $6 \mathrm{~mm}$ in agar-spot plates and showed a certain degree of activity against E. coli, Salmonella enteritidis, Salmonella Typhimurium and C. glabrata as well. In general, few food-borne yeasts have been found to possess clear antagonistic activity (Binetti et al., 2013; Silva et al., 2011). On the other hand, isolates with antimicrobial activity against food-borne pathogens may be helpful in ensuring the hygienic quality of dairy products, if used as adjunct cultures.

Table 5

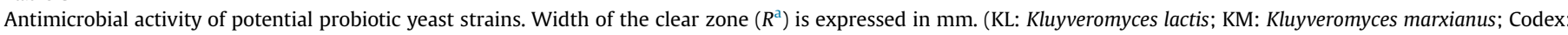
Saccharomyces cerevisiae).

\begin{tabular}{|c|c|c|c|c|c|c|c|c|c|}
\hline \multirow[t]{2}{*}{ Strains } & \multicolumn{9}{|l|}{ Indicators } \\
\hline & $\begin{array}{l}\text { C. glabrata } \\
\text { ATCC } 2001\end{array}$ & $\begin{array}{l}\text { C. parapsilosis } \\
\text { ATCC } 22019\end{array}$ & $\begin{array}{l}\text { C. albicans } \\
\text { ATCC } 10231\end{array}$ & $\begin{array}{l}\text { S. cerevisiae } \\
\text { ATCC } 9763\end{array}$ & $\begin{array}{l}\text { S. aureus ATCC } \\
25923\end{array}$ & $\begin{array}{l}\text { E. coli ATCC } \\
35150\end{array}$ & $\begin{array}{l}\text { S. enteritidis } \\
\text { ATCC } 13076\end{array}$ & $\begin{array}{l}\text { S. Typhimurium } \\
\text { ATCC14028 }\end{array}$ & $\begin{array}{l}\text { L. monocitogenes } \\
\text { ATCC } 7644\end{array}$ \\
\hline CODEXSB1 & 2 & - & - & - & 10 & 1 & 1 & 1 & - \\
\hline KEFIRKL1 & - & - & - & - & - & - & - & - & - \\
\hline 17bKL1 & 1 & - & - & - & 10 & 2 & 1.5 & 1 & - \\
\hline 17bKL2 & 1 & - & - & - & 8 & 1.5 & 1 & 1 & - \\
\hline NS2KL9 & 1 & - & - & - & 10 & 3 & 3 & 2 & - \\
\hline NS1KM2 & 1 & - & - & - & 2 & - & - & - & - \\
\hline 14KM1 & 1 & - & - & - & 1 & - & - & - & - \\
\hline 6688KM & 2 & - & - & - & 2 & - & - & - & - \\
\hline
\end{tabular}

$(-)$ no inhibition.

${ }^{\text {a }} R=(d$ Inhib - dSpot $) / 2$, where $d$ Inhib is the diameter of the zone without pathogen growth and $d$ Spot is the diameter of the spot. 
Table 6

Antimycotic susceptibility and haemolytic activity of yeast strains. (KL: Kluyveromyces lactis; KM: Kluyveromyces marxianus; Codex: Saccharomyces cerevisiae).

\begin{tabular}{|c|c|c|c|c|c|c|c|c|}
\hline \multirow[t]{2}{*}{ Strains } & \multicolumn{8}{|c|}{ Antimycotic ${ }^{a}$} \\
\hline & ITRAC & VOR1 & AMPH & CASP5 & KETOC & FLU & FLUCZ & B-haemolysis \\
\hline CODEXSB1 & S & $\mathrm{S}$ & $\mathrm{S}$ & $\mathrm{S}$ & $\mathrm{S}$ & S & S & - \\
\hline KEFIRKL1 & $\mathrm{S}$ & $\mathrm{S}$ & $\mathrm{S}$ & $\mathrm{S}$ & $\mathrm{S}$ & S & S & - \\
\hline 17bKL1 & $\mathrm{S}$ & $\mathrm{S}$ & S & $\mathrm{S}$ & $\mathrm{S}$ & S & S & - \\
\hline 17bKL2 & S & $\mathrm{S}$ & $\mathrm{S}$ & $\mathrm{S}$ & $\mathrm{S}$ & S & S & - \\
\hline NS2KL9 & $\mathrm{S}$ & S & $\mathrm{S}$ & $\mathrm{S}$ & $\mathrm{S}$ & $S$ & S & - \\
\hline NS1KM2 & $\mathrm{S}$ & $\mathrm{S}$ & S & $\mathrm{S}$ & $\mathrm{S}$ & $\mathrm{S}$ & S & - \\
\hline 14KM1 & $\mathrm{S}$ & $\mathrm{S}$ & S & $\mathrm{S}$ & $\mathrm{S}$ & $\mathrm{S}$ & $\mathrm{S}$ & - \\
\hline 6688KM & $\mathrm{S}$ & $S$ & S & $\mathrm{S}$ & $\mathrm{S}$ & $\mathrm{S}$ & S & - \\
\hline
\end{tabular}

(S) Susceptible (-) no activity.

a Itraconazole (ITRAC, $8 \mu \mathrm{g}):>23 \mathrm{~mm}(\mathrm{~S}), 22-14 \mathrm{~mm}(\mathrm{I}), \leq 13 \mathrm{~mm}(\mathrm{R})$; Voriconazole (VOR1, $1 \mu \mathrm{g})$ : $\geq 17 \mathrm{~mm}(\mathrm{~S}), 16-14 \mathrm{~mm}(\mathrm{I}), \leq 13 \mathrm{~mm}(\mathrm{R})$; Amphotericin B (AMPH, $10 \mu \mathrm{g})$ :

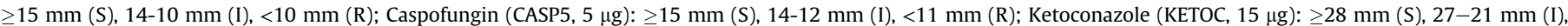
$\leq 20 \mathrm{~mm}(\mathrm{R})$; Flucytosine (FLU, $1 \mu \mathrm{g}):>20 \mathrm{~mm}(\mathrm{~S}), 19-12 \mathrm{~mm}(\mathrm{I}), \leq 11 \mathrm{~mm}(\mathrm{R})$; Fluconazole (FLUCZ, $25 \mu \mathrm{g}):>19 \mathrm{~mm}(\mathrm{~S}), 18-15 \mathrm{~mm}(\mathrm{I}), \leq 14 \mathrm{~mm}(\mathrm{R})$.

\subsection{Safety assessment}

In order for a strain to be used as probiotic culture, it should be evaluated for the presence of virulence determinants to assess what potential risks might be involved in its use. All the strains were non-haemolytic and sensitive to the clinical antimycotic agents tested (Table 6), in agreement with the findings of Ceugniez, Drider, Jacques, and Coucheney (2015) for Kluyveromyces dairy strains. Moreover, the natural yeast resistance to antibacterial antibiotics and the lack of genetic material transfer between bacteria and yeasts makes the latter good probiotic candidates (Czerucka et al., 2007).

\section{Conclusions}

In conclusion, the results presented in this study indicate that six yeast strains isolated from artisanal Fiore Sardo cheese possess interesting functional traits and absence of undesirable properties, and therefore could be considered appropriate probiotic candidates. Since the same strains have been shown to possess interesting technological characteristics in a previous study (Fadda et al., 2004), they could be used as adjunct cultures for contributing to the quality and health related functional properties of dairy products. However further studies need to be carried out on the evaluation of additional important features such as antioxidant activity, assimilation of cholesterol, anti-inflammatory properties, as well as for the validation of the isolates through clinical trials.

\section{Acknowledgements}

This work has been supported by Regione Autonoma Sardegna (L.R. 7/2007, CRP 17617). The authors thank Dr. Monica Deiana (Department of Biomedical Sciences, University of Cagliari, Italy) for providing the Caco- 2 cells.

\section{References}

Al-Seraih, A., Flahaut, C., Krier, F., Cudennec, B., \& Drider, D. (2015). Characterization of Candida famata isolated from poultry feces for possible probiotic applications. Probiotics and Antimicrobial Proteins, 7, 233-241.

Aloglu, H. S., Ozer, E. G. \& Oner, Z. (2016). Assimilation of cholesterol and probiotic characterization of yeast strains isolated from raw milk and fermented foods. International Journal of Dairy Technology, 69, 63-70.

Begley, M., Hill, C., \& Gahan, C. G. M. (2006). Bile salt hydrolase activity in probiotics. Applied and Environmental Microbiology, 72, 1729-1738.

Binetti, A., Carrasco, M., Reinheimer, J., \& Suarez, V. (2013). Yeasts from autochthonal cheese starters: Technological and functional properties. Journal of Applied Microbiology, 1-11.

Ceugniez, A., Drider, D., Jacques, P., \& Coucheney, F. (2015). Yeast diversity in a traditional French cheese "Tomme d'orchies" reveals infrequent and frequent species with associated benefits. Food Microbiology, 52, 177-184.
Clinical and Laboratory Standards Institute. (2009). Method for antifungal disk diffusion susceptibility testing of yeasts; approved guideline, 2nd ed., M44-A2. Wayne, PA: Clinical and Laboratory Standards Institute.

Corzo, G., \& Gilliland, S. E. (1999). Bile salt hydrolase activity of three strains of Lactobacillus acidophilus. Journal of Dairy Science, 82, 472-480.

Czerucka, D., Piche, T., \& Rampal, P. (2007). Review article: Yeast as probiotics Saccharomyces boulardii. Alimentary Pharmacology \& Therapeutics, 26, 767-778.

Del Re, B., Sgorbati, B., Miglioli, M., \& Palenzona, D. (2000). Adhesion, autoaggregation and hydrophobicity of 13 strains of Bifidobacterium longum. Letters in Applied Microbiology, 31, 438-442.

Diosma, G., Romanin, D. E., Rey-Burrusco, M. F., Londero, A., \& Garrote, G. L. (2014). Yeasts from kefir grains: Isolation, identification, and probiotic characterization. World Journal of Microbiology and Biotechnology, 30, 40-53.

El-Sharoud, W. M., Belloch, C., Peris, D., \& Querol, A. (2009). Molecular identification of yeasts associated with traditional Egyptian dairy products. Journal of Food Science, 74, M341-M356.

European Food Safety Authority. (2013). Scientific opinion on the maintenance of the list of QPS biological agents intentionally added to food and feed (2013 update). The EFSA Journal, 11, 1-106.

Fadda, M. E., Mossa, V., Pisano, M. B., Deplano, M., \& Cosentino, S. (2004). Occurrence and characterization of yeasts isolated from artisanal Fiore Sardo cheese. International Journal of Food Microbiology, 95, 51-59.

Fadda, M. E., Pisano, M. B., Scaccabarozzi, L., Mossa, V., Deplano, M., Moroni, P., et al. (2013). Use of PCR-restriction fragment length polymorphism analysis for identification of yeast species isolated from bovine intramammary infection. Journal of Dairy Science, 96, 1-6.

Fadda, M. E., Viale, S., Deplano, M., Pisano, M. B., \& Cosentino, S. (2010). Characterization of yeast population and molecular fingerprinting of Candida zeylanoides isolated from goat's milk collected in Sardinia. International Journal of Food Microbiology, 136, 376-380.

FAO/WHO. (2006). Probiotics in Food: Health and nutritional properties and guidelines for evaluation, FAO food and nutrition paper no. 85. Rome, Italy: FAO/WHO.

Fleet, G. H. (2007). Yeasts in food and beverages: Impact on product quality and safety. Current Opinion in Biotechnology, 18, 170-175.

Gueimonde, M., \& Salminen, S. (2006). New method for selecting and evaluating probiotics. Digestive Liver Disease, 38, S242-S247.

Jankowska, A., Laubiz, D., Antushevich, H., Zabielski, R., \& Grzesiuk, E. (2008). Competition of Lactobacillus paracasei with Salmonella enterica for adhesion to Caco-2 cells. Journal of Biomedicine and Biotechnology, 6, 2008. http://dx.doi.org/ 10.1155/2008/357964. Article ID 357964

Kumura, H., Tanoue, Y., Tsukahara, M., Tanaka, T., \& Shimazaki, K. (2004). Screening of dairy yeast strains for probiotic applications. Journal of Dairy Science, 87, 4050-4056.

Maccaferri, S., Candela, M., Centanni, M., Severgnini, M., Consolandi, C., et al. (2012). IBS-associated phylogenetic unbalances of the intestinal microbiota are not reverted by probiotic supplementation. Gut Microbes, 3, 406-413.

Maccaferri, S., Klinder, A., Brigidi, P., Cavina, P., \& Costabile, A. (2012). Potential probiotic Kluyveromyces marxianus B0399 modulates the immune response in Caco-2 cells and peripheral blood mononuclear cells and impacts the human gut microbiota in an in vitro colonic model system. Applied and Environmental Microbiology, 78, 956-964.

Moslehi-Jenebian, S., Pedersen, L. L., \& Jespersen, L. (2010). Beneficial effects of probiotic and food borne yeasts on human health. Nutrients, 2, 449-473.

Nguyen, T. D. T., Kang, J. H., \& Lee, M. S. (2007). Characterization of Lactobacillus plantarum $\mathrm{PHO4}$, a potential probiotic bacterium with cholesterol-lowering effects. International Journal of Food Microbiology, 113, 358-361.

Noriega, L., Cuevas, I., Margolles, A., \& de los Reyes-Gavilán, C. G. (2006). Deconjugation and bile salts hydrolase activity by Bifidobacterium strains with acquired resistance to bile. International Dairy Journal, 16, 850-855.

Pennacchia, C. Blaiotta, G. Pepe, O., \& Villani, F. (2008). Isolation of Saccharomyces cerevisiae strains from different food matrices and their preliminary selection for a potential use as probiotics. Journal of Applied Microbiology, 105, 1919-1928. 
Perricone, M., Bevilacqua, A., Corbo, M. R., \& Sinigaglia, M. (2014). Technological characterization and probiotic traits of yeasts isolated from Altamura sourdough to select promising microorganisms as functional starter cultures for cereal-based products. Food Microbiology, 38, 26-35.

Pisano, M. B., Viale, S., Conti, S., Fadda, M. E., Deplano, M., Melis, et al. (2014) Preliminary evaluation of probiotic properties of Lactobacillus strains isolated from Sardinian dairy products. BioMed Research International, 9. http:// dx.doi.org/10.1155/2014/286390. Article ID 286390.

Rahman, M. M., Kim, W. S., Kumura, H., \& Shimazaki, K. (2008). Autoaggregation and surface hydrophobicity. World Journal of Microbiology and Biotechnology, 24, 1593-1598.

Ramasamy, K., Abdullah, N., Wong, M. C. V. L., Karuthan, C., \& Ho, Y. W. (2010). Bile salt deconjugation and cholesterol removal from media by Lactobacillus strains used as probiotics in chickens. Journal of the Science of Food and Agriculture, 90, 65-69.

Romanin, D. F Serradell, M. Gonzalez Maciel, D, Lausada, N Garrote, G. L. \& Rumbo, M. (2010). Downregulation of intestinal epithelial innate response by probiotic yeasts isolated from kefir. International Journal of Food Microbiology, $140,102-108$.

Schillinger, U., \& Lücke, F. K. (1989). Antibacterial activity of Lactobacillus sake isolated from meat. Applied and Environmental Microbiology, 55, 1901-1906.

Silva, T., Reto, M., Sol, M., Peito, A., Peres, C. M., Prese, C., et al. (2011). Characterization of yeasts from Portuguese brined olives, with a focus on their potentially probiotic behavior. LWT - Food Science and Technology, 44, 1349-1354.

de Smet, I., van Hoorde, L., van de Woestyne, M., Christiaens, H., \& Verstraete, W. (1995). Significance of bile salt hydrolytic activities of lactobacilli. Journal of Applied Bacteriology, 79, 292-301.

Sourabh, A., Kanwar, S. S., \& Sharma, O. P. (2011). Screening of indigenous yeast isolates obtained from traditional fermented foods of western Himalayas for probiotic attributes. Journal of Yeast and Fungal Research, 2, 11-126.

Tiago, F. C. P., Martins, F. S., Souza, E. L. S., Pimenta, P. F. P., Araujo, H. R. C., Castro, I. M., et al. (2012). Adhesion to the yeast cell surface as a mechanism for trapping pathogenic bacteria by Saccharomyces probiotics. Journal of Medical Microbiology, 61, 1194-1207.

Tuomola, E., Crittenden, R., Playne, M., Isolauri, E., \& Salminen, S. (2001). Quality assurance criteria for probiotic bacteria. American Journal of Clinical Nutrition, $73,3935-398 S$

van der Aa Kühle, A., Skovgaard, K., \& Jespersen, L. (2005). In vitro screening of probiotic properties of Saccharomyces cerevisiae var. boulardii and food-borne Saccharomyces cerevisiae strains. International Journal of Food Microbiology. 101, 29-39.

Vizoso Pinto, M. G., Franz, C. M. A. P., Schillinger, U., \& Holzapfel, W. H. (2006) Lactobacillus spp. with in vitro probiotic properties from human faeces and traditional fermented products. International Journal of Food Microbiology, 109 205-214. 